\title{
USE OF ONTOlogies In Monitoring Mental Retardation ReHabilitation Process
}

\author{
LOVRENCIC, S.; VIDACEK-HAINS, V. \& KIRINIC, V.
}

Abstract: During treatment and rehabilitation process of children with mild mental retardation and associated disorders there is various data that has to be gathered and analysed, such as personal and family data, diagnosis, behaviour and prognosis. Additionally, in this paper are explained reasons and made initial efforts to add their usage of information and communications technologies to rehabilitation evaluation data. Ontologies, as modern knowledge bases, have already been developed for various domains, including the field of psychology. For a purpose of monitoring mental retardation rehabilitation process, possible use of ontologies is also explored. Ontology has been developed in Web Ontology Language using Protégé-OWL tool.

Key words: ontology, mental retardation, rehabilitation process, assistive technology
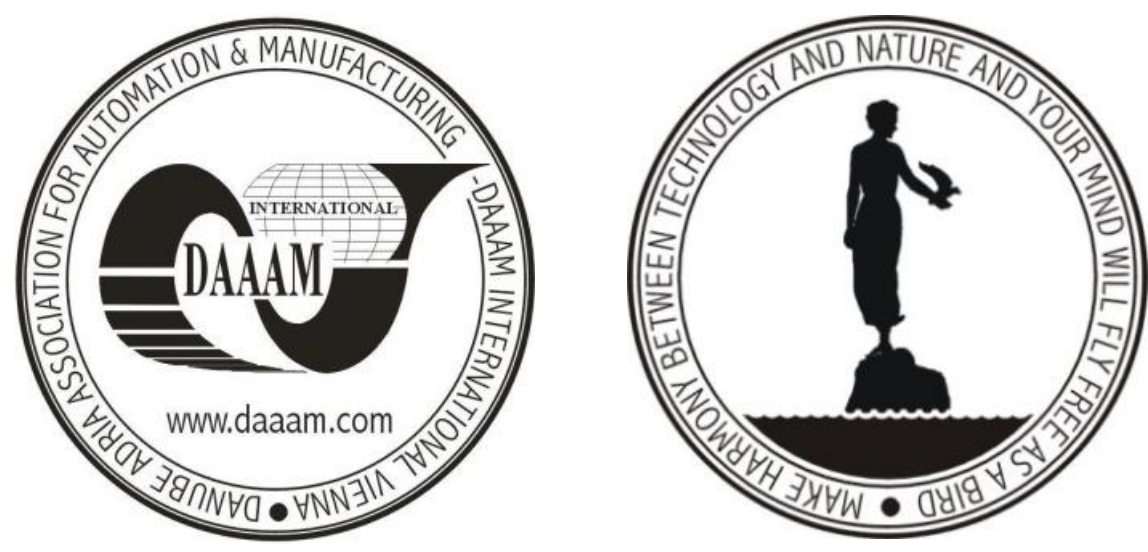

Authors' data: Dr.sc. Lovrencic, S[andra]; Dr.Sc. Vidacek-Hains, V[ioleta]; Dr.sc. Kirinic, V[alentina], University of Zagreb, Faculty of organization and informatics, Pavlinska2, HR-42000, Varazdin, Croatia, sandra.lovrencic@foi.hr, violeta.vidacekhains@foi.hr, valentina.kirinic@foi.hr

This Publication has to be referred as: Lovrencic, S[andra]; Vidacek-Hains V[ioleta] \& Kirinic, V[alentina] (2010). Use of Ontologies in Monitoring Mental Retardation Rehabilitation Process, Chapter 28 in DAAAM International Scientific Book 2010, pp. 289-300, B. Katalinic (Ed.), Published by DAAAM International, ISBN 978-3-901509-74-2, ISSN 1726-9687, Vienna, Austria

DOI: $10.2507 /$ daaam.scibook.2010.28 
Lovrencic, S.; Vidacek - Hains, V. \& Kirinic, V.: Use of Ontologies in Monitoring ...

\section{Introduction}

\subsection{Mental Retardation Rehabilitation}

According to World Health Organization (WHO, 2006), a mental health is a "state of well-being in which every individual realizes his or her own potential, can cope with the normal stresses of life, can work productively and fruitfully, and is able to make a contribution to her or his community". Mental retardation or intellectual disability is the type of disorder with insufficient quotient of intelligence (IQ), often associated with the behavioural problems and other dysfunctions like speech disorders, dyslexia, dysgraphia etc. Classification of mental disorders is contained within the DSM-IV classification - Diagnostic and Statistical Manual of Mental Disorders, issued by the American Psychiatric Association (APA, 2000). The borderline intellectual functioning quotient of intelligence is from 67 to 83 , mild mental retardation has IQ from 50-66, IQ of the moderate mental retardation is 33-49, IQ for the severe mental retardation is 16-32 and IQ for the profound mental retardation is below 16. Mental retardation affects about $1-3 \%$ of the population.

The most common cognitive/developmental assessment tools for children used by psychological clinical practitioners are Wechsler Intelligence Scale for Children WISC-IV) (Wechsler, 1991) and Developmental test of Intelligence of Children RTC (Cuturic, 2001). Because the instruments were constructed at the end of the last century, both of them have had several revisions. Children with mental retardation need specific types of rehabilitation or a process "aimed at enabling them to reach and maintain their optimal physical, sensory, intellectual, psychological and social functional levels" (WHO, 2006). According to the WHO, rehabilitation provides children with tools they need to attain independence and self-determination.

\subsection{Use of Assistive Technology}

An important role in helping the people with different kinds of disabilities to overcome some difficulties in their education (Dell et al., 2007), employment (de Jonge et al., 2006) and everyday life (Standen \& Brown, 2005; Davies et al., 2003) plays assistive technology. As defined in The Individuals with Disabilities Education Act Amendments of 1997, the assistive technology device is "any item, piece of equipment, or product system, whether acquired commercially off the shelf, modified, or customized, that is used to increase, maintain, or improve the functional capabilities of a child with a disability" (IDEA, 2004).

Assistive technology (AT) can be categorized in AT for vision, communication, access, hearing and AT for learning and studying (NCRL). Because of accessibility and affordability of computers there is a growing tendency for functions of certain assistive technology devices to be fully or partially realized by means of computers, either through hardware or software. Multiple functions of AT emphasize the importance of introducing computer and computer literacy to children with various disabilities. For this reason it is important to include factors that refer to their familiarity to and use of information and communication technologies (ICT) into rehabilitation process analysis. 
Semantic Web technologies, with ontologies as their central part, are nowadays widely used. They represent possibility to add semantics to information on World Wide Web (WWW), aiding in their better search, exchange, use and reuse. Although many aspects of Semantic Web yet have to be developed, a promise of better information management resulted in a large number of domains already represented with ontologies.

In a domain of psychology a model-oriented ontology that represents a multifactor personality model of Costa \& McCrae (Vidacek-Hains et al., 2007) has been developed, as well as children information literacy model ontology - CILMO (Kirinic et. al., 2008). The later was not only model-oriented but included actual survey data. In the more specific field of mental health, a work has been done in the usage of ontologies as assistive technology for improving user interaction with personal information management system for people with special needs (Karim \& Tjoa, 2006).Recent work provides also general directions how to design various systems, including ontologies, with purpose to aid mental health care treatment (Doherty et al., 2010). More general work proposes mental health ontology with classifications of disorders, causes and possible treatments (Hadzic et. al., 2008) and the other proposes a model for general mental health ontology including various client information (Coyle \& Doherty, 2008).

The main aim of presented research is to find out the possibilities for usage of ontologies in monitoring the success of the mental retardation rehabilitation process.

The first objective of this paper is to expand the use of ontologies in mental health care domain with specific information connected to children rehabilitation process in the mental rehabilitation institution using classic monitoring factors with inclusion of ICT usage.

The second objective is to explore how ontologies can assist to special teachers, educators, psychologists and other specialists in monitoring mental retardation rehabilitation process.

Possible limitations of the research could be ICT competencies of special teachers, psychologists and other professionals in usage ontology tools.

\section{Methodology}

For the purpose of monitoring the success of the rehabilitation process of children and youth with mental retardation and related developmental disorders, the Rehabilitation Process Monitoring Questionnaire RPMQ was constructed. The aim of this instrument is to give special teachers, educators, psychologists and other specialiststhe opportunity for data collecting and monitoring the rehabilitation process.

The instrument Rehabilitation Process Monitoring Questionnaire RPMQ contains 55 items, mostly with the multiple choice questions (Vidacek-Hains, 1998). The first part contains items linked to the demographic characteristic of children/users, second part contains medical diagnosis data and third part is linked to the user's family. The content of the fourth part of the RPMQ are the details of the 
Lovrencic, S.; Vidacek - Hains, V. \& Kirinic, V.: Use of Ontologies in Monitoring ...

process of rehabilitation and part five contains the criteria variables linked to the assessment of the rehabilitation success. Two criteria variables linked to ICT usage are added to part four - ICT usage rate and ICT usage skillfulness. The criteria of the success are the degree of the education, employment and family status.

The rehabilitation process was monitored by special teachers, educators, psychologists and other specialists, who completed the RPMQ instrument for users (7-21 years old). Users are children and youth with the specific needs, particularly children and youth with intellectual and related disorders. Majority of the users have diagnoses of mild mental retardation and related disorders like speech problems, dyslexia, dysgraphia, minimal cerebral dysfunction, behavioral problems etc.

\section{Ontologyfor monitoring mental retardationrehabilitation process}

\subsection{General Features of Ontologies}

Term ontology was taken from the field of philosophy almost 20 years ago and nowadays is commonly used in information science. Ontologies have become modern knowledge bases that are used to describe concepts and their hierarchical relationship, as well as concept attributes and their constraints (Gómez Pérez et. al., 2004). With the appearance of the concept of Semantic Web more than 10 years ago (Berners-Lee et. al., 2001) ontologies have found a central place in its realization. Semantically tagged information on WWW is expected to be achieved through the use of various technologies, as can be seen in Fig. 1 that represents a popular Semantic Web Layer Cake (W3C Semantic Web Activity, 2007).Each layer is built on top of another but top three layers - Proof, Trust and User interface \& Applications, as well as cryptography are currently only a vision.

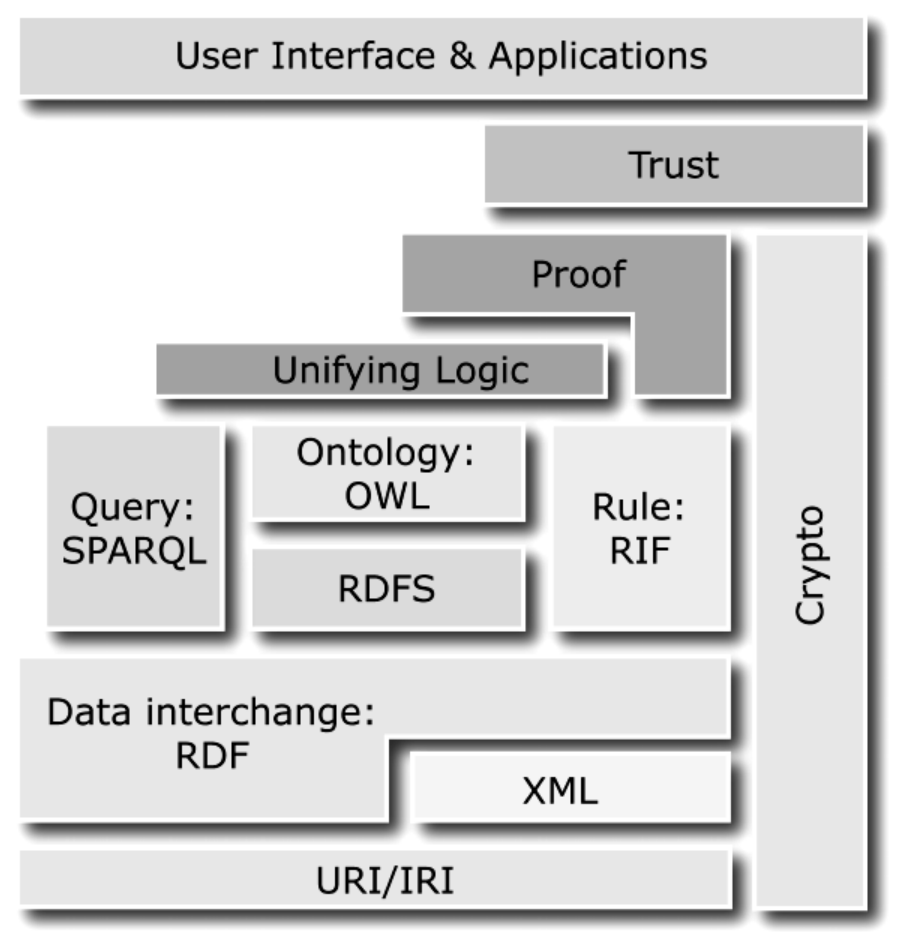

Fig. 1. Semantic Web Layer Cake 
A primary language chosen for ontologies is Ontology Web Language (OWL). It is designed to allow various statements about concepts and their attributes. The language itself is an upgrade on lower Semantic Web Layer Cake vocabularies (XML - eXtensibleMarkup Language, RDF - Resource Description Framework and RDFS - RDF Schema). Its expressiveness allows the usage of statements in formal logic, namely description logics (DL), which is a knowledge representation formalism that enables logical reasoning and conclusions about ontology individuals (concrete information stored in ontology). Extensive information about Semantic Web technologies, ontologies in particular, can be found in numerous literature (Antoniou \& van Hermelen, 2008; Hitzler et al., 2009).

There are several serializations for writing OWL ontologies, as well as tools that enable users to develop them graphically. One of most popular and most supported open source tools is Protégé (Stanford Center for Biomedical Informatics Research, 2010). It allows development of ontologies in two ways. First is the use of frames and first order logic (one of the first ontology development methods), which was used in previous work connected to mental health disorders (Vidacek-Hains et al., 2007) and information literacy (Kirinic et. al., 2008). Second is the use of OWL and DL that opens possibilities towards further inclusion of Semantic Web technologies and is used for development of ontology for monitoring mental retardation rehabilitation process.

\subsection{Ontology Description}

Ontology developed has a main class User that uses a number ofdatatype properties. Those properties describe a relationship between an individual (a specific user) and a data value. For example, a data type property named has Address is of type string and should store information about user address.

More specific for OWL ontology, it contains object properties that describe a relationship between two individuals. First individual is a specific user and the other is some kind of information about him. This information represents individuals of other classes; for example, class Institution has three subclasses: MentalHealthHospital, MentalRehailitationInstitution and SocialWorkCentre. Any user can have a relationship to an individual (a concrete hospital) of class MentalHealthHospital via object property called hasMentalHealthHospital. Upper levels of class hierarchy can be seen in Fig. 2.

Apart from directly describing the structure of the domain, it is also possible to use classes in a different way. They can represent value partitions that help in better description of main ontology classes and are actually design patterns (solutions how to model repeating problems). Concretely, this means that some information about users can be described with virtual individuals of assistant classes. If there is a class attribute with a finite number of possible values, it is not described as a data type property, but as a class with individuals that represent those values. It is also necessary to define that this class can have only listed individuals with a restriction that explicitly cites all of them (and is called enumerated class). In ontology for monitoring mental retardation rehabilitation process there is a number of such classes 
Lovrencic, S.; Vidacek - Hains, V. \& Kirinic, V.: Use of Ontologies in Monitoring ... and they are all subclasses of a class UserData. Enumerated class PracticalActivitiesAdoption can be seen in Fig. 3.

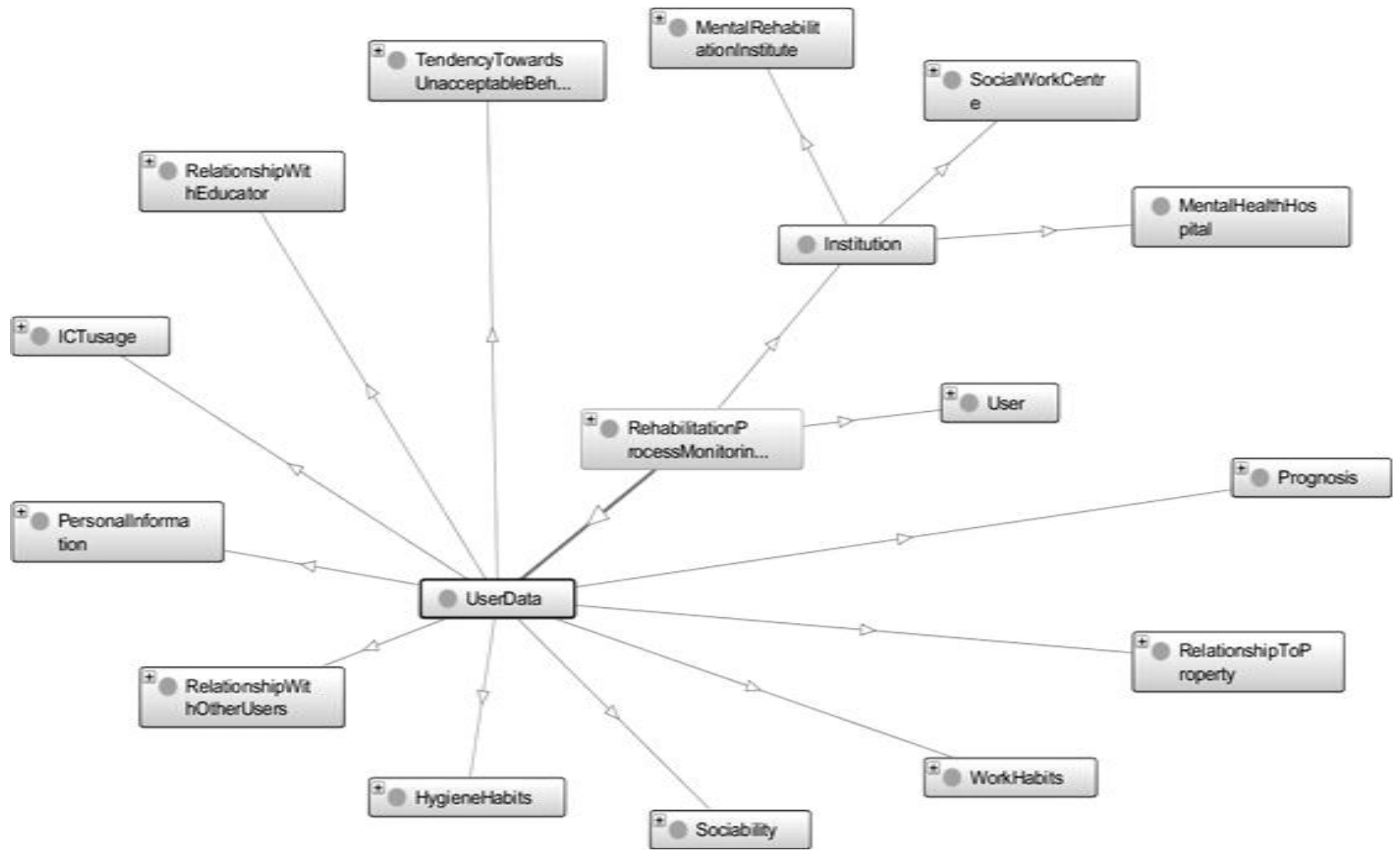

Fig. 2. Upper levels of ontology class hierarchy

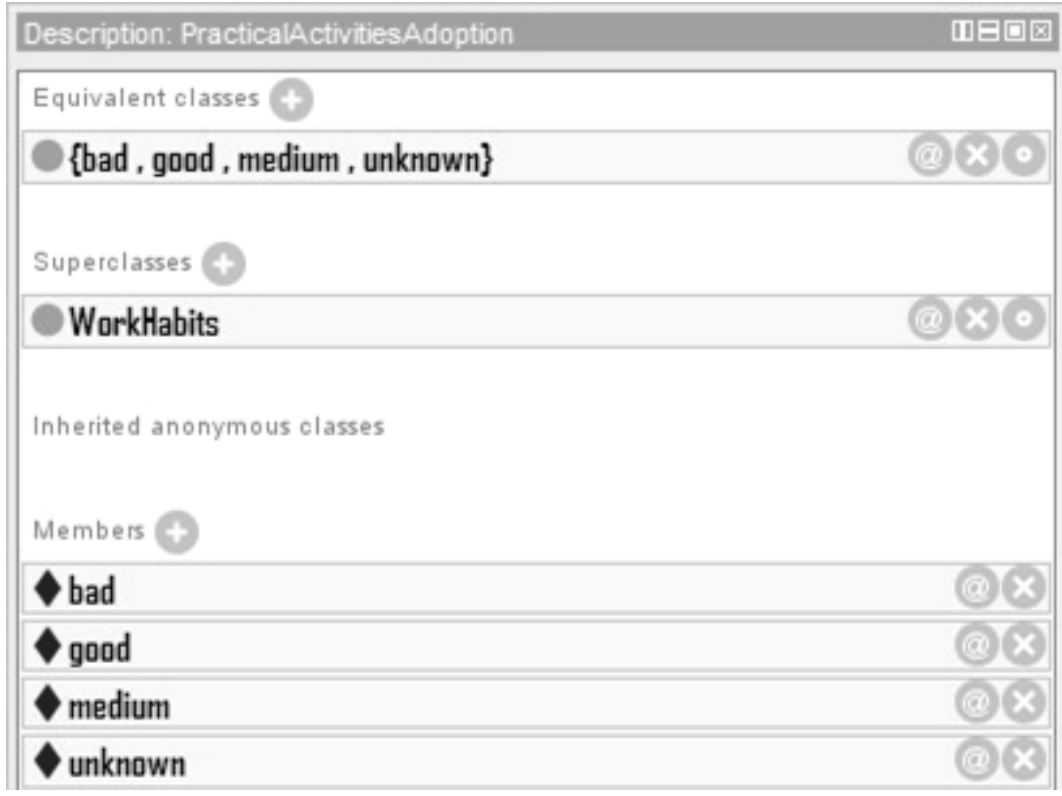

Fig. 3. Enumerated class PracticalActivitiesAdoption

Assistant class PracticalActivitiesAdoption is a subclass of classes UserData/WorkHabits. This class can have only individuals good, medium, bad and unknown. An object attribute hasPracticalActivitiesAdoption is created, as well as a restriction on class User that explicitly states that class User "hasPracticalActivitiesAdoption exactly 1PracticalActivitiesAdoption". This means that any individual of class User can have a relationship with only one individual of 
class PracticalActivitiesAdoptionvia object attribute hasPracticalActivitiesAdoption. Part of restrictions on class User can be seen in Fig. 4.

\begin{tabular}{|c|c|c|}
\hline Description: User & \multicolumn{2}{|c|}{ 由曰回圆 } \\
\hline OhasMentalRehabilitationlnstitution sume MentalRehabilitationlnstitute & $(0 \times 0$ & $\Delta$ \\
\hline OhasMotherOccupation same string & (0) $\times 0$ & \\
\hline hasName sume string & (0) $\times 0$ & \\
\hline OhasNumberOFSiblings exactly 1 nonNegativelnteger & (6) $\times 0$ & \\
\hline hasDrganicBrainDamage exactly 1 boulean & (a) $\times 0$ & \\
\hline OhasDrientationandDegree exactly 1 OrientationAndDegree & & \\
\hline OhasParentsSocial-PsychiatricPatology exactly 1 ParentsSocial-PsychiatricPatology & (a) $\times 0$ & \\
\hline OhasPedagogicalPreventionReaction exactly I PedagogicalPreventionReaction & (0) & \\
\hline OhasPedagogicalStimulationReaction exactly I PedagogicalStimulationReaction & (a) $\times 0$ & \\
\hline OhasPersanaHygieneHabitsAdaption exactly I PersanalHygieneHabitsAdaption & $x \odot$ & \\
\hline OhasPracticalActivitiesAdoption exactly I PracticalActivitiesAdoption & (1) $\times 0$ & \\
\hline hasPriorInstitution exactly I PriorInstitution & (6) $\times 0$ & \\
\hline hasDualitativeFamily Integrity exactly 1 QualitativeFamily Integrity & $(0 \times 0)$ & 7 \\
\hline
\end{tabular}

Fig. 4. Restrictions on class User

After ontology development, information about concrete individuals (users) can be entered. From Fig. 5 can be seen that original tool user interface is not well adjusted for people that are not well acquainted with information technology, such as special teachers, educators, psychologists and other specialists and this part of ontology still requires a certain amount of work.

\begin{tabular}{|c|c|c|}
\hline Property assertions: mm & 四田 & \\
\hline Object property assertions + & & - \\
\hline -hasICTusageSkilness bad & (c) $\times 0$ & \\
\hline -hasSocializationHabitsAdaption good & (6) $\times 3$ & \\
\hline$\square$ haslCTusageRate sometimes & (a) $\times 0$ & \\
\hline - hasPersonaHygieneHabitsAdaption medium & (a) $\times 0$ & \\
\hline$\square$ hasGeneralTidiness tidy & (6) $\times 0$ & \\
\hline$\square$ hasCammunicationSkillsAdaptionRate medium & (c) $\times 0$ & \\
\hline Data property assertions $\oplus$ & & \\
\hline hasDrganicBrainDamage false & $\times 0$ & \\
\hline - hasDiagnosis "ADHD" & (a) $\times 0$ & \\
\hline hasDiagnosis "mild mental retardation" & (a) $\times 0$ & \\
\hline hasNumberOfSiblings "2"^^nonNegativelnteger & (6) $\times 0$ & \\
\hline -hasCitizenship "Croatian" & (6) $\times 0$ & $\nabla$ \\
\hline
\end{tabular}

Fig. 5. Example of user data 
Lovrencic, S.; Vidacek - Hains, V. \& Kirinic, V.: Use of Ontologies in Monitoring ...

\section{Possibilitiesforapplication}

\subsection{Application in Mental Health Rehabilitation Monitoring}

Education is important part of rehabilitation for children with intellectual and related developmental disorders (Kirinic et al., 2010). Evaluation of the rehabilitation process of children with mental retardation and other difficulties is an important part of the successful special education. The RPMQ Questionnaire was firstly applied at the Institution for the rehabilitation of children with the mild mental retardation. The results of the research showed that users with multiple diagnoses from destroyed families have less success in the process of rehabilitation (Vidacek-Hains, 1998).

There are other possibilities for usage of the RPMQ questionnaire, for example:

- as a feedback to the social workers from Centres for social work who made the decision of sending children to the institutions;

- in Institutions for rehabilitation;

- in Schools for children with special needs.

Data analysis could be improved in several ways by the rehabilitation process monitoring ontology supported by Protégé-OWL tool:

- use of ontology as a knowledge base, similarly as proposed mental health ontology (Hadzic et. al., 2008; Coyle \& Doherty, 2008) can enable storage of large amount of various data;

- a querying system that gives filtered data access can enable quick access to various information;

- easy modification with new criteria variables and restrictions ensures that eventual changes will be entered with minimal effort;

- expansion with various Semantic Web technologies offers a wide range of possibilities for future use.

\subsection{Inclusion of Assistive Technology Evaluation Criteria}

To enhance the educational process the computer can be used to tutor or to explore, can be applied as a tool, used to communicate, and also used for assessment and management purposes (Means, 1994 in Florian, 2004). Using computers in tuition is known as computer-assisted instruction (CAI) which, according to Means (Means, 1994 in Florian, 2004), "help teachers individualize learning and learners to work at their own pace". The second use of the computer is to build exploratory learning environments allowing "pupils to interact with the material and have more control over their learning" and "emphasize exploration as opposed to drill and practice or the reinforcement of skills and knowledge".

Applied as a tool, the computer (computer literacy) represents a prerequisite for other types of learning with technology, and is thus essential for everyday life beyond school. The computer together with e.g. voice synthesizers and voice recognition software is a very important and powerful assistive technology device helping children with disability to communicate too. 
Finally, the computer/ICT can be used as a tool for assessment - in diagnosing the nature of the child's learning difficulty, and as a management tool, e.g. to develop individual education plans (IEPs) adapted to each student.

There are some special benefits of using computers in education. Compared to traditional education, computer-aided education has proved to be more effective and efficient, primarily owing to additional motivation enhanced by the interaction with the computer.

Apart from motivation, using computers in the education of persons with developmental disorders is important as it contains the social interaction component. For instance, parents and teachers of children with attention deficit hyperactivity disorder (ADHD) have noticed that computer-aided collaborative work was fairly effective in developing social skills. Tan and Cheung (Tan \& Cheung, 2008) assume that the reason for this may be that children who are normally not so popular among their peers are likely to be better accepted by their peers on account of their computer skills.

One of the goals of special education is the acquisition of specific independent living skills. Standen and Brown (Standen \& Brown, 2005) give an overview of using virtual reality in rehabilitation of persons with intellectual disabilities, both children and adults. Their paper comprises studies utilizing virtual technology to promote skills for independent living: grocery shopping, preparing food, orientation, road safety and manufacturing skills.

The computer has been established as a useful tool for helping children enhance their linguistic abilities, most notably reading and writing. Compared with human teaching, the computer has obvious advantages such as endless repetition, direct feedback, and the fact that children do not feel judged (Segers \&Verhoeven, 2005). The computer can help e.g. in teaching sight word recognition to young children with developmental disabilities (Lee \& Vail, 2005), and to help acquisition of writing spelling in particular - e.g. Word Prediction Software (Mirenda \& Turoldo, 2006).

Because of numerous stated benefits of the usage of ICT as assistive technology in rehabilitation of mental retardation, it would be advisable to expand criteria variables for ICT usage with other criteria that can be extracted from above examples. Those criteria variables can be connected to specific diagnoses and data analysis would show what kind of assistive technology can be used with certain types of mental retardation and other disorders.

\section{Conclusionand future research}

There is a strong focus on development of various assistive technology systems, including ontologies, with a purpose to help in rehabilitation process of people with mental retardation. Ontology presented in this paper is, on the other side, trying to help special teachers, educators, psychologists and other specialists involved in the process of rehabilitation of children with mental retardation and other disabilities. It is shown that information vital for analysis of this process can be easily presented as ontological knowledge. The main obstacle is that the original tool user interface is not well adjusted for people that are not well acquainted with information technology. 
It is also elaborated why is very important to evaluate users familiarity and usage of ICT. Initial general criteria variables for ICT usage - ICT usage rate and ICT skilfulness are added. They show general information about possibilities for usage of ICT in rehabilitation process of children with mental retardation.

As can be seen from previous section, further work in this field offers various possibilities. The development of intuitive user interface is of main concern for ontology to be used in real environment. Nevertheless, the main interests are to connect created ontology with other developed models that have information about diagnoses and treatments and to further expand the evaluation criteria in two ways:

- monitoring rehabilitation process for more mental health disorders;

- monitoring differences in possible use of ICT for various disorders.

First expansion means inclusion of other relevant information important when analyzing rehabilitation process for various disorders, because the initial model (Rehabilitation Process Monitoring Questionnaire RPMQ) is created for mild mental retardation and associated disorders. This is currently in focus because results from research described in this paper will be used to apply the ontology model for monitoring the rehabilitation processes for different types of disorders (for example visual impaired or deft children, children with psychiatric sicknesses or even children with specific disorders like dyslexia, dysgraphia or dyscalculia).

Second expansion is more important and can enable development of appropriate assistive technology systems to aid in mental retardation rehabilitation for a specific set of disorders. Depending on disorder type, possibilities of children to use ICT technologies vary and in this way rehabilitation results for methods that use ICT tools can be enhanced.

\section{References}

APA - American Psychiatric Organization (2000). Diagnostic and Statistical Manual of Mental Disorders, Fourth Edition (DSM IV TR), Office of Publishing Operator American Psychiatric Organization, ISBN 089042-0246-6, Washington DC

Antoniou, G. \& van Hermelen, F. (2008). A Semantic Web Primer (second edition), The MIT Press, ISBN: 978-0-262-01242-3, Cambridge

Berners-Lee, T.; Hendler, J. \& Lassila, O. (2001). The Semantic Web. Scientific American, Vol. 284, No. 5, May 2001, pp. 29-37, ISSN: 0036-8733

Coyle, D. \& Doherty, G. (2008). Towards Ontologies for Technology in Mental Health Interventions, Proceedings of First International Workshop on Ontologies in Interactive Systems (ONTORACT'08), Bouamrane, M-M.; Luz, S.\&Masodian, M. (Eds.), pp. 18-26,ISBN: 978-1-4244-3459-6, Liverpool, September 2008, IEEE, Washington

Cuturic, N. (2001). Psiho motorički razvoj djeteta u prve dvije godine života s normama razvoja (Psychomotoric child development in first two years with development norms), Naklada Slap, Jastrebarsko, ISBN 953-191-056-1

Davies, D.K.; Stock, S.E. \&Wehmeyer M.L. (2003). Application of computer simulation to teach ATM access to individuals with intellectual disabilities. 
Education and Training in Developmental Disabilities, Vol. 38, No. 4, 2003, pp. 451-456, ISSN: 1547-0350

De Jonge, D.; Scherer, M.J. \&Rodger, S. (2006). Assistive Technology in the Workplace, Mosby-Elsevier Health Sciences, ISBN: 0-323-04130-2, St. Louis

Dell, A.G.; Newton, D. \& Petroff, J. (2007). Assistive Technology in the Classroom: Enhancing the School Experiences of Students with Disabilities, Prentice Hall, ISBN: 0131191640

Doherty, G.; Coyle, D. \& Matthews, M. (2010). Design and evaluation guidelines for mental health technologies. Interacting with Computers, Vol. 22, No. 4, July 2010, pp. 243-252, ISSN: 0953-5438

Florian, L. (2004). Uses Of Technology That Support Pupils With Special Educational Needs" in ICT and Special Educational Needs, A Tool for Inclusion, (ed. Lani Florian and John Hegarty), McGraw-Hill International, 2004., Available from: http://www.mcgraw-hill.co.uk/openup/chapters/ 033521195X.pdf Accesed on: 2010-06-30

Gómez Pérez, A.; Fernández-López, V. \& Corcho, O. (2004). Ontological Engineering, Springer-Verlag, ISBN 1-85233-551-3, London, UK

Hadzic, M.; Chen, M. \& Dillon, T. S. (2008). Towards the mental health ontology,In Proceedings of the IEEE international conference on bioinformatics and biomedicine (BIBM 2008), Xue-wen, C. \& Sun, K. (Eds.), pp. 284-288, ISBN: 978-0-7695-3452-7, Philadelphia, November 2008, IEEE, Washington

Hitzler, P.; Krötzsch, M. \& Rudolph, S. (2009). Foundations of Semantic Web Technologies, CRC Press, ISBN: 978-1-4200-9050-5, Boca Raton

IDEA 2004 (2004). Individuals with Disabilities Education Improvement Act of 2004, Available from: http://idea.ed.gov/download/statute.html Accesed on: 2010-06-30

Karim, S. \&Toja A. M. (2006). Towards the Use of Ontologies for Improving User Interaction for People with Special Needs, Proceedings of $10^{\text {th }}$ International Conference on Computers Helping People with Special Needs (ICCHP 2006), Miesenberger, K.; Klaus, J.; Zagler, W. \& Karshmer, A. (Eds.),pp. 77-84 , ISBN: 978-3-540-36020-9, Linz, July 2006,Springer, Berlin

Kirinic, V., Vidacek-Hains, V., Kovacic, A. (2010). Computers in Education of Children with Intellectual and RelatedDevelopmental Disorders. International Journal of Emerging Technologies in Learning (iJET) Vol. 5, No. 2, p.p. 12-16. Available from:http://online-journals.org/i-jet/article/view/1246 Accessed: 2010-05-25

Kirinic, V.; Vidacek-Hains, V. \& Lovrencic, S. (2008). CILMO - Children's Information Literacy Model Ontology, Chapter 37 in DAAAM International Scientific Book 2008, B. Katalinic (Ed.), pp. 371-386, DAAAM International, ISBN 78-3-901509-66-7, Vienna, Austria

Lee, Y. \& Vail, C.O. (2005). Computer-based reading instruction for young children with disabilities. Journal of Special Education Technology, Vol. 20, No.1, 2005, pp. 5-18, ISSN: 0162-6434 Available from: http://jset.unlv.edu/20/ JSETv20n1.pdf Accesed on: 2008-11-25 
Means, B. (ed.) (1994).Technology and Education Reform: The Reality Behind the Promise, Jossey-Bass, ISBN: 1555426255, San Francisco

Mirenda, P. \& Turoldo, K. (2006). The Impact of Word Prediction Software on the Written Output of Students with Physical Disabilities. Journal of Special Education Technology, Vol. 21, No.1, 2006, pp. 5-12, ISSN: 0162-6434 Available from: http://jset.unlv.edu/20/JSETv21n3.pdf\#page $=6$ Accesed on: 2009-02-1

NCRL - North Central Regional Educational Laboratory.Assistive Technology to Meet K-12 Student Needs, Available from: http://www.ncrel.org/sdrs/areas/ issues/methods/technlgy/te7assist.htm Accesed on: 2010-06-30

Segers, E. \& Verhoeven, L. (2005). Long-term effects of computer training of phonological awareness in kindergarten. Journal of Computer Assisted Learning, Vol. 21, Issue 1, 2005, pp.17-27, ISSN: 0266-4909

Standen, P. \& Brown, D. (2005). Virtual Reality in the Rehabilitation of People with Intellectual Disabilities: Review. CyberPsychology\&Behaviour, Vol. 8, No. 3, 2005, pp. 272-282, ISSN: 2152-2715

Stanford Center for Biomedical Informatics Research (2010). What is Protégé?, Available from: http://protege.stanford.edu/overview/Accessed: 2010-05-11

Tan, T.S. \& Cheung, W.S. (2008). Effects of computer collaborative group work on peer acceptance of a junior pupil with attention deficit hyperactivity disorder (ADHD). Computers \& Education, Vol. 50, No. 3, 2008, pp. 725-741, ISSN:0360-1315

Vidacek-Hains, V. (1998). An evaluation the success of rehabilitation for children with mental retardation and other difficulties. Journal of $2^{\text {nd }}$ International Conference Mental Health of People with Difficulties in Social Integration, Videc, K. (Ed.), p.p. 178-184, ISBN 953-96760-2-0, Trakoscan, May 1989, Croatian Association of Special Teachers, Zagreb

Vidacek-Hains, V.; Lovrencic, S. \& Kirinic, V. (2007). Personality Model Representation using Ontology, Chapter 37 in DAAAM International Scientific Book 2007, B. Katalinic (Ed.), pp. 423-434, DAAAM International, ISBN 3901509-60-7, Vienna, Austria

Wechsler, D. (1991). WICS IV Clinical Assessment and Intervention : Wechsler Intelligence Scale for Children, Elsevier Inc., ISBN 987-0-12-3736-0,San Diego, CA

WHO - World Health Organization (2006). Disabilityand Rehabilitation WHO Action Plan 2006-2011, Available from: http://www.who.int/disabilities/ publications/dar_action_plan_2006to2011.pdf Accessed: 2010-05-25

W3C Semantic Web Activity (2007). Latest "layercake" diagram, Available from: http://www.w3.org/2007/03/layerCake-small.png Accesed on: 2010-06-21 